\title{
Sociedad Española de Cirugía de la Mano (SECMA). 40 años de historia (1969-2009)
}

\author{
Dr. A. Lluch HomedeS

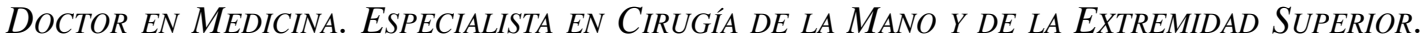 \\ MIEMBRO DEL INSTITUT KAPLAN \\ Correspondencia: \\ Dr. Alberto Lluch Homedes \\ Institut Kaplan \\ Paseo Bonanova, 9 \\ 08022 Barcelona \\ e-mail: albertolluch@institut-kaplan.com
}

$\mathrm{E}$ n sus inicios, tanto en España como en la mayoría de los países europeos, la Cirugía de la Mano la realizaban los cirujanos generales, los cuales trataban principalmente infecciones y lesiones traumáticas agudas. Durante la guerra civil española (19361939) los procedimientos quirúrgicos más frecuentemente practicados eran los desbridamientos de tejidos necróticos o infectados y las amputaciones.

Sterling Bunnell (1882-1957) era un cirujano general que ejercía en San Francisco, California. Se le considera como el padre de la Cirugía de la Mano, que según él debía aunar las especialidades de traumatología, cirugía plástica y neurocirugía. La publicación de su libro Surgery of the Hand en el año 1944 propició que sus conocimientos fuesen accesibles a otros cirujanos que tuviesen interés en la Cirugía de la Mano y de la Extremidad Superior, pues como muy bien dijo: mecánicamente la mano comienza en el codo, pero funcionalmente empieza en el hemisferio cerebral opuesto.

En las décadas de los años 1950 y 1960, la mayor parte de las enseñanzas de la Cirugía de la Mano en España la aportaron nuestros colegas franceses, algunos de cuales habían visitado a Sterling Bunnell en los Estados Unidos. Dichos médicos franceses fueron invitados pa- ra dar conferencias en Cursos y Seminarios sobre Cirugía de la Mano en España. Algunos médicos españoles también tuvieron la oportunidad de realizar estancias, durante períodos cortos de tiempo, en hospitales franceses. Los Médicos españoles tenían grandes dificultades para viajar a los EEUU durante aquellos años, tanto por la precaria situación económica ocasionada por la Guerra civil, como por el aislamiento político al no haber participado España en la II Guerra Mundial. También se debe tener en cuenta que el inglés era un idioma minoritariamente hablado. Todas estas circunstancias supusieron una barrera importante para la adquisición de conocimientos médicos por los médicos españoles. Tuvieron que esperar a que los libros escritos en alemán y principalmente inglés, fuesen traducidos al castellano.

Georg Hohmann publicó uno de los primeros libros sobre la cirugía de la mano en Europa, el cual fue traducido al castellano en el año1955 con el título de Mano y Antebrazo. Durante los primeros años de la década de los sesenta tuvo lugar en España un creciente interés en la Cirugía de la Mano, principalmente propiciado por la traducción al castellano del libro escrito por Sterling Bunnell La Cirugía de la Mano. Le siguieron otras publicaciones, también traducidas al castellano, como Lesiones 
Traumáticas de la Mano por R. Souquet y A.R. Chancholle, Lesiones Tendinosas en los Traumatismos de la Mano por J. Michon y R. Vilain y Tratamiento Quirúrgico de Urgencia en los Traumatismos de la Mano por R. Vilain y J.F. Dupuis. El libro Traité de Chirurgie de la Main, escrito por François Iselin no fue traducido castellano, pero podía ser fácilmente leído por la mayoría de los cirujanos españoles, quienes habían aprendido el francés como una segunda lengua durante sus estudios.

En España, el primero en escribir un libro sobre la patología de la mano fue Mario Oliveras, médico rehabilitador del Instituto de Inválidos de Carabanchel, que publicó Traumatismos e Incapacidades de la Mano, en el año 1948. Otros pioneros fueron Eugenio T. López-Trigo Torres, del Hospital Provincial de Valencia, que en el año 1948 publicó Lesiones Osteoarticulares Traumáticas de la Muñeca, y E. Velilla Mateo, del Hospital de la Princesa de Madrid, que en el año 1950 publicó Traumatología y Cirugía Reparadora de la Mano. Más recientemente, Carlos Irisarri publicó en el año 1987 la monografía Cirugía de la Mano Traumática.

Durante el transcurso de los años, comenzaban a diferenciarse las distintas especialidades quirúrgicas, como la Cirugía Ortopédica y Traumatología y la Cirugía Plástica y Reconstructiva, ambas interesadas en el diagnóstico y tratamiento de la patología de la mano. La Primera Reunión Nacional sobre la Cirugía de la Mano la organizó el Dr. Alfredo Quintana Montero, cirujano Ortopédico y Traumatólogo de Zaragoza, en febrero de 1969. Como ponentes fueron invitados los Drs. Pulvertaff, Vainio, Tubiana y Souquet. También participaron otros cirujanos de la mano españoles, como los Drs. A. Santos Palazzi-Duarte, Vicente Senti Montagut, Jaime Planas y Fernando Henríquez de Salamanaca. El Dr. A. Santos Palazzi-Duarte, cirujano ortopédico de Barcelona había realizado los primeros injertos de tendones flexores en la década de los años 1940. El Dr. Jaime Planas, también de Barcelona, era un cirujano plástico experto en la reparación de lesiones de los tendones flexores de los dedos y en la cobertura de los defectos cutáneos en la mano. El Dr. Vicente Senti-Montagut de Ma- drid se especializó en la cirugía de la Mano Reumatoide, sobre la cual escribió una monografía. El Dr. Fernando Henriquez de Salamanaca era un cirujano plástico de Madrid, incansable orador y profesor, que fue reconocido como un gran maestro en la patología de la musculatura intrínseca y en el tratamiento de la mano paralítica.

El creciente interés en la cirugía de la mano y el entusiasmo generado por la reunión en Zaragoza, propició que el 11 de mayo de 1969 se crease la Sociedad Española de Cirugía de la Mano (SECMA). Los Miembros fundadores fueron: Fernando Enríquez de Salamanca, quien fue el primer Presidente, Tomás Antona, José Antonio Bañuelos, Antonio Bengoa, José María Cañadell, Ricardo de Manuel, Manuel Masoliver, Ramón Moreno, Ángel Santos Palazzi-Duarte, Jaime Planas, Alfredo Quintana, Ignacio Sampera y Vicente Sentí. La Sociedad fue fundada con 29 Miembros.

La segunda Reunión Nacional de Cirugía de la Mano se celebró en Barcelona en noviembre de 1973, y fue co-presidida por los Drs. Bañuelos y Planas. La tercera Reunión Nacional se celebró en Zaragoza en junio de 1974, asistiendo más de doscientos cirujanos, y presidida por Dr. Alfredo Quintana, quien invitó a los Drs. Yves Allieu, José Cantero, A. Chancholle, N. Haimovici, Ian Matev, Enzo Morelli, Aldo de Negri, A. Pannike y Wynn Parry.

En 1975 se decidió que la SECMA debería celebrar un Congreso bianual en diferentes ciudades españolas. A continuación se expone un listado de los Congresos que se han celebrado:

I. Bilbao, 1975 (Rafael Uribe)

II. Barcelona, 1977 (Jaime Planas). Conjunto con la Sociedad Italiana

III. Oviedo, 1979 (Ricardo de Manuel)

IV. Valencia, 1981 (Vicente Mirabet)

V. Zaragoza, 1983 (Alfredo Quintana)

VI. Sitges, Barcelona, 1985 (Juan Oller). Conjunto con las Sociedades Francesas de Cirugía de la Mano y de Microcirugía

VII. Murcia, 1987 (Francisco Pedreño)

VIII. Platja d'Aro, Girona, 1989. (Fernando Fonseca). Conjunto con la Sociedad Italiana 
IX. Madrid, 1991 (Carlos Irisarri). Conjunto con las Sociedades Americana y Francesa

X. Vigo, 1993 (Estanislao Troncoso). Conjunto con la Sociedades Brasileña y Argentina

XI. Barcelona, 1995 (Alberto Lluch)

XII. Gandía, Valencia, 1997 (Ignacio Soria). Conjunto con la Sociedad Alemana de Cirugía de la Mano

XIII. Las Palmas de Gran Canaria, 1999 (José Antonio Medina)

XIV. Sevilla 2001 (Pilar Pradilla). Conjunta con los IV Encuentros Iberoamericanos

XV. Pamplona, 2003 (Santiago Amillo)

XVII. Valladolid 2005 (Miguel Ángel Martín Ferrero). Conjunto con la Sociedad Británica

XVII. Zaragoza, 2007 (Gustavo García Julve). Conjunto con las Sociedades de los Países Europeos del Este de Europa, y VII Encuentro Iberoamericano

XVIII. Cádiz, 2009 (Javier Noya Gómez)

La SECMA también ha sido invitada a participar en Reuniones y Congresos organizados por otras Sociedades Nacionales extranjeras. El primero tuvo lugar en Montpellier (Francia) en mayo de 1975, con la participación de la Sociedad Francesa (Dr. Rabischong) y la Sociedad Italiana (Dr. Bonola). Durante esta Reunión, el Prof. Bonola propuso la creación de una Federación de Sociedades de Cirugía de la mano de origen latino, que sería el germen de lo que ahora es la Federación Europea de Sociedades de Cirugía de la Mano (FESSH). En mayo de 1996 tuvo lugar en Cascais (Portugal) una reunión conjunta con la Sociedad Portuguesa, y en septiembre del año 2002 un Congreso conjunto con la Sociedad Italiana en la ciudad de Florencia (Italia).

Bajo el liderazgo del Dr. Carlos Irisarri, la SECMA auspició el realizar Encuentros IberoLatino-Americanos de Cirugía de Cirugía de la Mano, conjuntamente o intercalados con el Congreso bianual que celebra la SECMA. Se expone a continuación el listado de los Congresos que se han celebrado:

I. Playa Bávaro, República Dominicana. (Marcos Núñez y Héctor Herranz). Abril 1998.
II. Sao Paulo, Brasil (Walter Albertoni). Noviembre 1999.

III. Cancún, México (Alejandro Espinosa). Mayo 2002.

IV. Sevilla (Pilar Pradilla). Mayo 2001.

V. Florencia, Italia (Máximo Cerusso y Michele Arienzo). Septiembre 2002.

VI. Joinville, Brasil (Valdir Steglich y Flavio Faloppa). 30 marzo a 2 abril 2005.

VII. Zaragoza (Gustavo García Julve). Junio 2007.

VIII. Buenos Aires, Argentina (Miguel Capomassi). Octubre 2007.

IX. Porto, Portugal (Dr. José Manuel Amarante). Abril 2008.

X. Cartagena de Indias, Colombia (Dr. F. Camacho). Octubre 2009.

En noviembre de 1974, los doctores Ballester y Borrell fundaron la primera Unidad especializada en Cirugía de la Mano en el Hospital de la Cruz Roja de Hospitalet, Barcelona.

Al final de los años setenta y a principios de los ochenta, se desarrolló un interés creciente en la microcirugía y la cirugía de reimplante, lo cual dio a luz a la creación centros especializados en microcirugía, principalmente en Madrid, Zaragoza, Burgos, Cuenca, Barcelona y Sabadell. Resultante de este impulso fue la creación de un Centro Nacional de Emergencia de la Mano en el año1981.

Un jalón en la Historia del SECMA fue la creación de la Revista de Cirugía de la Mano en el año 1973. Fue fundada y dirigida por el Doctor Alfredo Quintana Montero, publicándose dos volúmenes al año, con algún suplemento extraordinario sobre temas específicos.

En el año 1998, siendo presidente el Dr. Higinio Ayala Palacios, y por iniciativa del Dr. Carlos Irisarri y posterior aprobación por la Asamblea, se inició un proyecto de renovación de la Revista Española de Cirugía de la Mano con el propósito de que se integrasen la Sociedad Portuguesa y otras Sociedades Iberoamericanas, y que se denominaría Revista Iberoamericana de Cirugía de la Mano. Inicialmente, dicha revista sería el órgano oficial de la Asociación Argentina de Cirugía de la Mano, la Sociedade Brasileira de Cirurgia da Mão, la Sociedade Portuguesa de Cirurgia da Mão y la Sección Mano de la Sociedad 
de Cirugía Plástica de Uruguay. El redactor-jefe fue el Dr. Alfredo Quintana Guitián, y los directores han sido sucesivamente los Drs. Xavier Mir, Francisco del Piñal y José María Arandes.

La SECMA fue Miembro Fundador de la Federación Internacional de Sociedades para la $\mathrm{Ci}$ rugía de la Mano (IFSSH) en el año 1969, y de la Federación Europea de Sociedades de Cirugía de la Mano (FESSH) en el año 1991. Los representantes de la SECMA en ambas Federaciones han sido los Drs. Fernando Enríquez de Salamanca, Juan Oller, Alberto Lluch, Marc García-Elías, José Antonio Medina y Enrique Mackenney.

La SECMA ha participado activamente en las decisiones de la IFSSH y de FESSH a través de sus delegados internacionales: Fernando Enríquez de Salamanca, Juan Oller, Alberto Lluch, Marc García-Elías, José Antonio Medina y Enrique Mckenney. En Junio del 2000 la SECMA organizó en Barcelona el VII Congreso de la Federación Europea de Sociedades de Cirugía de la Mano (FESSH) bajo la presidencia de Alberto LLuch.

En 1982, la SECMA inició unos diálogos con el Gobierno y la Universidad Españolas con la finalidad de reconocer a la Cirugía de la Mano como una especialidad independiente.
La última reunión con el Ministerio la mantuvo el Dr. García-Elías, como presidente de la SECMA, en 2006, recibiendo un trato muy favorable. De todas maneras, el reconocimiento de la especialidad todavía no se ha conseguido, y es probable que tengamos que esperar las decisiones tomadas por la UMES en Bruselas.

La participación de los cirujanos de la Mano españoles en los Congresos Internacionales se ha incrementado considerablemente durante los últimos años. Así pues, España fue el segundo país de Europa, tras Inglaterra, en número de participantes al congreso de la FESSH de Glasgow en junio del 2006. También debemos congratularnos de que varios miembros de la SECMA hayan ocupado cargos relevantes tanto en la FESSH (Alberto Lluch como tesorero, Marc García-Elías como SecretarioGeneral, Francisco del Piñal como Asistente Editor del Journal of Hand Surgery Europeo) como en la IFSSH (Alberto Lluch y Marc García-Elías como Miembros del Consejo Directivo).

De los 29 miembros fundadores de la SECMA en el año 1969, en el momento actual la Sociedad está formada por 351 Miembros: 6 Miembros de Honor, 22 Extranjeros, $205 \mathrm{Nu}-$ merarios, 104 Asociados y 14 Eméritos.
1. Irisarri Castro C. Notas sobre la Historia de la Sociedad Española de Cirugía de la Mano.

\section{BIBLIOGRAFÍA}

2. Quintana Montero A. Orígenes y evolución de la Sociedad Española de Cirugía de la Mano. Rev Esp Cir Mano 1997; 24: 59-80. 